\title{
Another plan for negation
}

\author{
Nissim Francez \\ Computer Science Department \\ The Technion-IIT \\ Haifa, Israel \\ francez@cs.technion.ac.il
}

\section{Introduction}

My point of departure is a recent defence of the "Australian Plan" for a paraconsistent negation by Berto and Restall in [1] against a recent attack by De and Omori in [4], defending the "American Plan". The heart of the controversy is whether to define paraconsistent negation model-theoretically by means of four-valued matrices (with gluts and gaps) or via frames of "points" (going also by the names 'situations', 'states', or 'cases' in general).

In this paper, I aim at presenting another plan, that shares some features with the Australian plan on the one hand, but radically departs from the latter on the other hand.

The central idea in the Australian plan is ([1], p. 3):

whether $\neg \varphi$ holds here depends on whether $\varphi$ holds elsewhere,

where 'here' refers to some point (of evaluation) and 'elsewhere' refers to evaluation at some other point. Thus, negation is rendered by the Australian plan as a point-shift operator, much like a modality with Kripke model-theory.

Australasian Journal of Logic (16:5) 2019, Article no. 1 
I want to adopt the shift-view of negation, but change the paradigm: instead of shifting points, shift formulas. Thus,

whether $\neg \varphi$ holds ("here") depends on whether a certain different $\psi$ holds ("here" too).

This paradigm makes "here" redundant, as it does to the whole idea of using frames of points.

Another idea of [1] I adopt is that negation is an exclusion operator, grounded on a primitive notion of incompatibility. This term has been used extensively in the literature, alas in different senses. For example, Restall [13] uses it as a relation between states (points) in an incompatibility frame, in the spirit of the Australian plan. Brandom [2] uses incompatibility as relation between sets of sentences and subsets of the power-set of sentences; and there are more.

In my plan the relata of incompatibility change: the are not associated with points, but are formulas and finite sets of simple formulas (conjunctions of atomic formulas) with an imposed primitive incompatibility on atomic sentences!

The key feature of my plan is not that it leads to paraconsistency, but rather that it leads to (a family of) contra-classical logics. But in contrast to other ways of obtaining contra-classicality, my plan obtains it without "loosing" classical validities, by avoiding closure under uniform substitution. Atomic formulas have an "absolute" deductive role.

My plan departs from standard conventions in yet another way. Usually, atomic formulas are considered independent of each other. In a model-theory based on valuations assigning truth-values to formulas, a valuation assigns a truth-value to an atomic formula independently of the truth-values it assigns to any other atomic formulas. According to my plan, there are dependencies among atomic formulas. In particular, (mutual) incompatibility is attributed by a model to (finite) collections of atomic formulas, constraining their truth-values. When used to define negation, the resulting negation is

Australasian Journal of Logic (16:5) 2019, Article no. 1 
not just excluding an assertion, but also suggesting the truth of another assertion, an incompatible alternative to the excluded assertion. This approach is particularly suitable for a view according to which formulas are a "behind the scene regimentations" of some natural language sentences, and their interdependency incorporates into the logic some material connections. In [6], this kind of negation is called ${ }^{1}$ conversational negation, and is traced back to its origins in the linguistics literature.

Note that I am referring continuously to atomic formulas, not to propositional variables, as the generators of compound formulas. Indeed, this view does not support uniform substitutions, that would not preserve relationships among atomic formulas imposed by my plan.

Applying such an approach, for example, to obtain a Relevance Logic might impose relevance of one atomic sentence to another one, going beyond the traditional syntactic property of variable sharing in defining relevant implication and relevant consequence.

\section{Atomic incompatibility bases}

In the sequel, I assume a propositional object language freely-generated by a set $P=\left\{p_{i} \mid i \geq 0\right\}$ of atomic formulas, ranged over by meta-variables $p, q$. For convenience, I assume the standard connectives: ' $\neg$ ' (negation), ' $\wedge$ ' (conjunction), ' $V$ ' (disjunction and ' $\rightarrow$ ' (conditional). I use $\varphi, \psi$ as metavariables ranging over formulas, $\alpha, \beta$ over conjunctions ${ }^{2}$ of atomic formulas and $\Gamma, \Delta$ - over finite sets of formulas.

Definition 2.1 (atomic incompatibility base) $A n$ atomic incompatibility base is a mapping $\mathbf{i}: P \Rightarrow \mathcal{P}_{f}(P) / \emptyset$ (i.e., the range of $\mathbf{i}$ consists of finite, non-empty subsets of atomic formulas), s.t.:

\footnotetext{
${ }^{1}$ The main interest in that paper is in computational means of identfying "plausible" alternatives to the negated sentence.

${ }^{2}$ Where convenient, a conjunction of atomic formulas is also considered as the finite set of its those atoms.
}

Australasian Journal of Logic (16:5) 2019, Article no. 1 


$$
\begin{gathered}
(\text { nref }) \quad p \notin \mathbf{i}(p) \\
(\text { sym) } p \in \mathbf{i}(q) \text { iff } q \in \mathbf{i}(p) \\
(\text { part) If } p \in \mathbf{i}(q) \text { then } \mathbf{i}(p)=(\mathbf{i}(q) /\{p\}) \cup\{q\}
\end{gathered}
$$

\section{Remarks:}

1. By definition, for every $p \in P, \mathbf{i}(p) \neq \emptyset$. Each atomic formula has at least one (other) atomic formula incompatible with it.

2. Incompatibility is irreflexive, symmetric and transitive.

3. The mapping $\mathbf{i}$ partitions $P$ into incompatibility classes s.t. for every incompatibility class $I C$ : if $p, q \in I C$ then $p \in \mathbf{i}(q)$ (and, hence, $q \in \mathbf{i}(p))$. Clearly, incompatibility classes are not equivalence classes, as incompatibility is not reflexive.

The intention is that truth-values are assigned in such a way that in every incompatibility class there is exactly one true member.

To direct the thought, the reader may think of a generic incompatibility class as a representation of colours $^{3}$, where atomic formulas regiment sentences assigning a colour to some specific object, say o. Clearly, o can have exactly one colour (all over).

Next, the mapping $\mathbf{i}$ is extended to $\hat{\mathbf{i}}$, mapping also compound formulas. The range of $\hat{\mathbf{i}}$ consists of conjunctions of atomic formulas.

Definition 2.2 (proper conjunctions of atoms) A conjunction of atoms $\alpha=\wedge_{2 \leq j \leq m} p_{j}$ is $\mathbf{i}$-proper, denoted by $\pi(\alpha)$, if no $q_{j_{1}}$ and $q_{j_{2}}\left(\right.$ for $\left.j_{1} \neq j_{2}\right)$ are in the same $I C$ of $\mathbf{i}$.

\footnotetext{
${ }^{3}$ For simplicity, assume there are only five colours: red, green, blue, white and black.
} 
A conjunction of atoms $\alpha$ which is not proper is improper. The role of being proper will become clear in the sequel.

\section{Definition 2.3}

$$
\begin{aligned}
& \hat{\mathbf{i}}(p)=\{q \wedge \alpha \mid q\in \mathbf{i}(p), \pi(\alpha), p \notin \alpha\} \\
& \hat{\mathbf{i}}(\neg \varphi)=\bigcap_{\alpha \in \hat{\mathbf{i}}(\varphi)} \hat{\mathbf{i}}(\alpha) \\
& \hat{\mathbf{i}}(\varphi \wedge \psi)=\hat{\mathbf{i}}(\varphi) \cup \hat{\mathbf{i}}(\psi) \\
& \hat{\mathbf{i}}(\varphi \vee \psi)=\hat{\mathbf{i}}(\varphi) \cap \hat{\mathbf{i}}(\psi) \\
& \hat{\mathbf{i}}(\varphi \rightarrow \psi)=\hat{\mathbf{i}}(\neg \varphi) \cap \hat{\mathbf{i}}(\psi)
\end{aligned}
$$

Note that for every $\varphi, \hat{\mathbf{i}}(\varphi)$ contains only $\mathbf{i}$-proper atomic conjunctions.

Example 2.1 For better readability, suppose we have the mnemonicallynamed atomic formulas.

$$
\{\text { red, green, blue, short, long }\}
$$

Consider an atomic incompatibility base $\mathbf{i}_{0}$ satisfying

$$
\begin{gathered}
\mathbf{i}_{0}(\text { red })=\{\text { green }, \text { blue }\} \quad \mathbf{i}_{0}(\text { green })=\{\text { red }, \text { blue }\}, \quad \mathbf{i}_{0}(\text { blue })=\{\text { red }, \text { green }\} \\
\mathbf{i}_{0}(\text { short })=\{\text { long }\}, \mathbf{i}_{0}(\text { long })=\{\text { short }\}
\end{gathered}
$$

To avoid notational clutter and in anticipation of Definition 4.7, only relevant representatives of $\hat{\mathbf{i}}_{0}$ are shown. The conjunctions generated by (2.4) are displayed modulo their commutativity.

$$
\begin{gathered}
\hat{\mathbf{i}}(\text { red })=\begin{array}{c}
\{\text { blue } \wedge \text { short, green } \wedge \text { short }, \\
\text { blue } \wedge \text { long, green } \wedge \text { long }\}
\end{array} \\
\hat{\mathbf{i}}(\text { green })=\begin{array}{c}
\{\text { blue } \wedge \text { short }, \text { red } \wedge \text { short }, \\
\text { blue } \wedge \text { long, red } \wedge \text { long }\}
\end{array} \\
\hat{\mathbf{i}}(\text { blue })=\begin{array}{c}
\{\text { red } \wedge \text { short, green } \wedge \text { short }, \\
\text { red } d \wedge \text { long, green } \wedge \text { long }\}
\end{array}
\end{gathered}
$$

Australasian Journal of Logic (16:5) 2019, Article no. 1 


$$
\begin{gathered}
\hat{\mathbf{i}}(\text { long })=\{\text { blue } \wedge \text { short, green } \wedge \text { short }, \text { red } \wedge \text { short }\} \\
\hat{\mathbf{i}}(\text { short })=\{\text { blue } \wedge \text { long, green } \wedge \text { long, red } \wedge \text { long }\} \\
\hat{\mathbf{i}}_{0}(\text { red } \wedge \text { long })=\hat{\mathbf{i}}_{0}(\text { red }) \cup \hat{\mathbf{i}}_{0}(\text { long })=\begin{array}{c}
\{\text { blue } \wedge \text { short, green } \wedge \text { short }, \\
\text { blue } \wedge \text { long, green } \wedge \text { long }, \\
\text { red } \wedge \text { short }\}
\end{array} \\
\hat{\mathbf{i}}_{0}(\text { red } \vee \text { long })=\hat{\mathbf{i}}_{0}(\text { red }) \cap \hat{\mathbf{i}}_{0}(\text { long })=\{\text { blue } \wedge \text { short }, \text { green } \wedge \text { short }\} \\
\hat{\mathbf{i}}_{0}(\neg r e d)=\{\text { red } \wedge \text { short, red } \wedge \text { long }\} \\
\hat{\mathbf{i}}_{0}(\neg(\text { red } \wedge \text { long }))=\{\text { red } \wedge \text { long }\}
\end{gathered}
$$

From Definition (2.3) we have:

Corollary 1 (non-reflexivity) For every $\alpha$ : $\alpha \notin \hat{\mathbf{i}}(\alpha)$.

Proof. Assume, towards a contradiction, that for some $\alpha=q_{1} \wedge \cdots \wedge q_{l}, l \geq 1$

$$
\alpha \in \hat{\mathbf{i}}(\alpha)=\cup_{1 \leq i \leq l} \hat{\mathbf{i}}\left(q_{i}\right)
$$

W.l.o.g, assume $\alpha \in \hat{\mathbf{i}}\left(q_{1}\right)=\left\{p \wedge \beta \mid p \in \hat{\mathbf{i}}\left(q_{1}\right), q_{1} \notin \beta\right\}$.

But this is impossible, as $q_{1}$, a conjunct in $\alpha$, cannot be $p$ because $q_{1} \notin \mathbf{i}\left(q_{1}\right)$ and cannot be in $\beta$.

Corollary 2 (negation) For every $\varphi$

$$
\hat{\mathbf{i}}(\varphi) \cap \hat{\mathbf{i}}(\neg \varphi)=\emptyset
$$

Australasian Journal of Logic (16:5) 2019, Article no. 1 
Proof. Assume, towards a contradiction, that for some $\alpha$

$$
\alpha \in \hat{\mathbf{i}}(\varphi) \cap \hat{\mathbf{i}}(\neg \varphi)
$$

But $\hat{\mathbf{i}}(\neg \varphi)=\bigcap_{\beta \in \hat{\mathbf{i}}(\varphi)} \hat{\mathbf{i}}(\beta)$, and $\alpha$ is one of those $\beta$ s. Hence, $\alpha \in \hat{\mathbf{i}}(\alpha)$, contradicting the non-reflexivity Corollary 1.

\section{Truth and consequence}

The main point in the definition of truth (in a model) is that the atomic formulas in every incompatibility class are assigned a truth-value in a coordinated way, not independently of each other. In particular, some $p \in I C$ is assigned $f$ iff some $q \in \mathbf{i}(p)$ is assigned $t$.

Definition 3.4 (i-valuations) For a given atomic incompatibility base $\mathbf{i}$, a i-valuation $v$ is a a mapping $P \Rightarrow\{t, f\}$ s.t

$$
v \llbracket p \rrbracket= \begin{cases}t & \text { iff for every } q \in \mathbf{i}(p) v \llbracket q \rrbracket=f \\ f & \text { iff for some } q \in \mathbf{i}(p) v \llbracket q \rrbracket=t\end{cases}
$$

For example, the atomic formula, say $p$, expressing the proposition ' $o$ is red (all over)' is false iff one of the incompatible atomic formulas $q \in \mathbf{i}(p)$, expressing the proposition that $o$ has another colour (all over), is true.

A i-valuation is naturally extended to compound formulas very much like in familiar definitions, but with truth under a valuation depending "in the background" on the atomic incompatibility base $\mathbf{i}$.

$$
\begin{gathered}
v \llbracket \neg \varphi \rrbracket=t \text { iff } v \llbracket \varphi \rrbracket=f \\
v \llbracket \varphi \wedge \psi \rrbracket=t \text { iff } v \llbracket \varphi \rrbracket=t \text { and } v \llbracket \psi \rrbracket=t \\
v \llbracket \varphi \vee \psi \rrbracket=t \text { iff } v \llbracket \varphi \rrbracket=t \text { or } v \llbracket \psi \rrbracket=t \\
v \llbracket \varphi \rightarrow \psi \rrbracket=t \text { iff } v \llbracket \varphi \rrbracket=f \text { or } v \llbracket \psi \rrbracket=t
\end{gathered}
$$

An immediate corollary of (3.11) is that $v \llbracket \neg \neg \varphi \rrbracket=v \llbracket \varphi \rrbracket$.

Australasian Journal of Logic (16:5) 2019, Article no. 1 
The next theorem extends the dependency of truth on incompatible atomic sentences to compound formulas. It underlies the property of negation being not just excluding (the negated formula) but requiring the truth of one of the incompatible alternatives of the negated formula.

Definition 3.5 (truth characteristics) A set of conjunctions of atoms $\mathbf{j}(\varphi)$ is a truth characteristics (t-characteristics) for $\varphi$ under $\mathbf{i}$ iff for every $\mathbf{i}$ valuation $v$ :

$$
v \llbracket \varphi \rrbracket= \begin{cases}t & \text { iff for every } \alpha \in \mathbf{j}(\varphi) v \llbracket \alpha \rrbracket=f \\ f & \text { iff for some } \alpha \in \mathbf{j}(\varphi) v \llbracket \alpha \rrbracket=t\end{cases}
$$

Theorem 3.1 (truth and incompatibility) For every $\varphi$ and every $\mathbf{i}$-valuation $v, \hat{\mathbf{i}}(\varphi)$ is the $e^{4}$-characteristic for $\varphi$ under $\mathbf{i}$.

Spelled out in detail: For every $\varphi$ and every i-valuation $v$ :

$$
v \llbracket \varphi \rrbracket= \begin{cases}t & \text { iff for every } \alpha \in \hat{\mathbf{i}}(\varphi) v \llbracket \alpha \rrbracket=f \\ f & \text { iff for some } \alpha \in \hat{\mathbf{i}}(\varphi) v \llbracket \alpha \rrbracket=t\end{cases}
$$

Proof. The proof is by a simple induction on $\varphi$. I show the proof of one direction only, the proof of the other direction being similar. Fix an arbitrary i-valuation $v$.

Basis: The basis is when $\varphi=p$, an atomic formula, and is given by (3.10).

Step: Assume (3.1) holds for $\varphi_{i}, i=1,2$.

$$
\begin{aligned}
\varphi= & \varphi_{1} \wedge \varphi_{2}: \text { Suppose } v \llbracket \varphi \rrbracket=t . \text { By }(3.11), v \llbracket \varphi_{1} \rrbracket=t \text { and } v \llbracket \varphi_{2} \rrbracket=t . \\
& \text { By the induction hypothesis, for every } \alpha \in \hat{\mathbf{i}}\left(\varphi_{1}\right) v \llbracket \alpha \rrbracket=f \text { and for } \\
& \text { every } \alpha \in \hat{\mathbf{i}}\left(\varphi_{2}\right) v \llbracket \alpha \rrbracket=f . \text { Therefore, for every } \alpha \in \hat{\mathbf{i}}\left(\varphi_{1}\right) \cup \hat{\mathbf{i}}\left(\varphi_{2}\right) \\
& v \llbracket \alpha \rrbracket=f .
\end{aligned}
$$

\footnotetext{
${ }^{4}$ Uniqueness follows from the 'iff' in (3.12).
}

Australasian Journal of Logic (16:5) 2019, Article no. 1 
$\varphi=\varphi_{1} \vee \varphi_{2}:$ By (3.11), $v \llbracket \varphi_{1} \rrbracket=t$ or $v \llbracket \varphi_{2} \rrbracket=t$. W.l.o.g, assume the former. By the induction hypothesis, for every $\alpha \in \hat{\mathbf{i}}\left(\varphi_{1}\right) v \llbracket \alpha \rrbracket=f$. Therefore, for every $\alpha \in \hat{\mathbf{i}}\left(\varphi_{1}\right) \cap \hat{\mathbf{i}}\left(\varphi_{2}\right) v \llbracket \alpha \rrbracket=f$.

$\varphi=\varphi_{1} \rightarrow \varphi_{2}$ : By (3.11), $v \llbracket \varphi_{1} \rrbracket=f$ or $v \llbracket \varphi_{2} \rrbracket=t$.

$v \llbracket \varphi_{1} \rrbracket=f:$ By (3.11), $v \llbracket \neg \varphi_{1} \rrbracket=t$. By the induction hypothesis, for every $\alpha \in \hat{\mathbf{i}}\left(\neg \varphi_{1}\right) v \llbracket \alpha \rrbracket=f$. Therefore, for every $\alpha \in$ $\hat{\mathbf{i}}\left(\neg \varphi_{1}\right) \cap \hat{\mathbf{i}}\left(\varphi_{2}\right) v \llbracket \alpha \rrbracket=f$.

$v \llbracket \varphi_{2} \rrbracket=t$ : By the induction hypothesis, for every $\alpha \in \hat{\mathbf{i}}\left(\varphi_{2}\right) v \llbracket \alpha \rrbracket=$ $f$. Therefore, for every $\alpha \in \hat{\mathbf{i}}\left(\neg \varphi_{1}\right) \cap \hat{\mathbf{i}}\left(\varphi_{2}\right) v \llbracket \alpha \rrbracket=f$.

$\varphi=\neg \varphi_{1}$ : By (3.11), $v \llbracket \varphi_{1} \rrbracket=f$. By the induction hypothesis, for some $\alpha \in \hat{\mathbf{i}}\left(\varphi_{1}\right) v \llbracket \alpha \rrbracket=t$. Therefore, for every $\chi \in \hat{\mathbf{i}}(\alpha) v \llbracket \chi \rrbracket=f$, implying that for every $\chi \in \cap_{\alpha \in \hat{\mathbf{i}}\left(\varphi_{1}\right)} \hat{\mathbf{i}}(\alpha)=f$.

\section{Proposition 3.1 (double negation incompatibility)}

$$
\hat{\mathbf{i}}(\neg \neg \varphi)=\hat{\mathbf{i}}(\varphi)
$$

Proof. It is immediately seen that $\hat{\mathbf{i}}(\neg \neg \varphi)$ is a $t$-characteristics for $\varphi$ under $\mathbf{i}$ (and $\hat{\mathbf{i}}(\varphi)$ is a $t$-characteristics for $\neg \neg \varphi$ under $\mathbf{i}$ ) and so both are equal.

The definition of the model-theoretic notions of consequence and validity also resemble their usual definition, but they "hide" a different notion of truth and falsity in a model.

\section{Definition 3.6 (consequence, validity)}

i-consequence: For a given atomic incompatibility base $\mathbf{i}, \psi$ is a (logical) i-consequence of $\Gamma$, denoted $\Gamma=_{\mathbf{i}} \varphi$, iff for every $\mathbf{i}$-valuation $v: v \llbracket \psi \rrbracket=t$ whenever $v \llbracket \varphi \rrbracket=t$ for every $\varphi \in \Gamma$.

i-validity: $\varphi$ is $\mathbf{i}$-valid iff $\emptyset \mid={ }_{\mathbf{i}} \varphi$.

Australasian Journal of Logic (16:5) 2019, Article no. 1 
An immediate corollary of the definition of $\mathbf{i}$-consequence is the following.

Proposition 3.2 (Tarskian consequence relation) $\models_{\mathbf{i}}$ is a Tarskian consequence relation for every atomic compatibility base $\mathbf{i}$.

We can regard every atomic incompatibility base $\mathbf{i}$ and its associated consequence relation $\models_{\mathbf{i}}$ as inducing a contra-classical logic $\mathcal{L}_{i}$

Observation: Every i-valuation is also a classical valuation, but not vice versa. Therefore, every classical validity (a tautology) is by definition also $\mathbf{i}$-valid for every atomic compatibility base $\mathbf{i}$. However, there are classically contingent formulas (i.e. classical non-tautologies), that may be $\mathbf{i}$-valid for some $\mathbf{i}$, because the refuting classical valuation is not a $\mathbf{i}$-valuation for that $\mathbf{i}$ (see Example 3.2 below).

It is important to realise that not "loosing" classical tautologies does not contradict the post-completeness of classical logic, because each $\mathcal{L}_{\mathbf{i}}$ is not closed under uniform substitution, the latter being essential in the proof that adding validities to classical logic results in inconsistency.

By the same token, every classical contradiction is a $\mathbf{i}$-contradiction for every atomic incompatibility base $\mathbf{i}$. However, there are classically contingent formulas which are $\mathbf{i}$-contradictions for some atomic incompatibility base $\mathbf{i}$, because their satisfying classical valuations are not $\mathbf{i}$-valuations for that $\mathbf{i}$.

Thus, contra-classical logics are emerging from this definition of negation!

Example 3.2 Let $\varphi$ be $p \vee q$ for $p$, $q$ different atomic formulas. Clearly, $p \vee q$ is not classically valid. It is refuted by the classical valuation $\hat{v}$ by which both $\hat{v} \llbracket p \rrbracket=f$ and $\hat{v} \llbracket q \rrbracket=f$. Consider now an atomic compatibility base $\mathbf{i}$ where $\mathbf{i}(p)=\{q\}, \mathbf{i}(q)=\{p\}$. A i $\mathbf{i}$-valuation enforces $\hat{v} \llbracket p \rrbracket \neq \hat{v} \llbracket q \rrbracket$ and, therefore, $\hat{v}$ is not a $\mathbf{i}$-valuation. No $\mathbf{i}$-valuation can refute $p \vee q$, and so $\models_{\mathbf{i}} p \vee q$.

In addition, for the same $\mathbf{i}$,

$$
p \models_{\mathbf{i}} \neg q
$$

Australasian Journal of Logic (16:5) 2019, Article no. 1 
Again, this is not a classically valid consequence, but a refuting valuation is not a $\mathbf{i}$-valuation.

Similarly, $\neg(p \vee q)$, classically contingent, is a $\mathbf{i}$-contradiction for the above $\mathbf{i}$. It has no $\mathbf{i}$-valuation validating it.

Theorem 3.2 (i-validity characterisation) For every atomic compatibility base $\mathbf{i}$ :

$$
\models_{\mathbf{i}} \varphi \text { iff } \hat{\mathbf{i}}(\varphi)=\emptyset
$$

Proof.

only if: Suppose $\models_{\mathbf{i}} \varphi$, and assume, towards a contradiction, that $\alpha \in \hat{\mathbf{i}}(\varphi)$. Since $\alpha$ is $\mathbf{i}$-proper, for some $\mathbf{i}$-valuation $v v \llbracket \alpha \rrbracket=t$. But then, by (3.1) $v \llbracket \varphi \rrbracket=f$, contradicting the $\mathbf{i}$-validity of $\varphi$.

if: Suppose that $\hat{\mathbf{i}}(\varphi)=\emptyset$. Consider an arbitrary i-valuation $v$. By the emptiness assumption, it holds vacuously that $v \llbracket \alpha \rrbracket=f$ for every $\alpha \in$ $\hat{\mathbf{i}}(\varphi)$. Hence, by $(3.1), v \llbracket \varphi \rrbracket=t$. Since $v$ is arbitrary, $\varphi$ is $\mathbf{i}$-valid.

\section{Natural deduction for $\mathcal{L}_{\mathrm{i}}$}

In this section I introduce, for each $\mathbf{i}$, a natural deduction system $\mathcal{N}_{\mathbf{i}}$, capturing $\models_{\mathbf{i}}$. The idea is to use $\hat{\mathbf{i}}$ itself within the I/E-rules.

Remark: The presence of $\hat{\mathbf{i}}$ in the I/E-rules is analogous to the presence of worlds (and their accessibility relation) in natural-deduction systems for modal logics. See [7] and [12], and further references herein.

Australasian Journal of Logic (16:5) 2019, Article no. 1 


\subsection{Restricting $\hat{\mathrm{i}}$ to finite sets}

There is a difficulty of using $\hat{\mathbf{i}}$ in the proof-system, namely the fact that $\hat{\mathbf{i}}(\varphi)$ can, in general, contain infinitely many conjunctions of atoms. However, since $\varphi$ may have only finitely many atomic formulas as sub-formulas, only finitely many of the conjunctions in $\hat{\mathbf{i}}(\varphi)$ are relevant to its deductive role. So, the first step is to restrict $\hat{\mathbf{i}}(\varphi)$ to its finitely many relevant conjunctions.

Definition 4.7 (restricting $\hat{\mathbf{i}}$ ) Let $\hat{\mathbf{i}}_{r}(\varphi){ }^{d f} \cdot\{\alpha / \mathbf{a}(\varphi) \mid \alpha \in \hat{\mathbf{i}}(\varphi)\}$.

Clearly, $\hat{\mathbf{i}}_{r}(\varphi)$ is finite for every $\varphi$. Furthermore, $\hat{\mathbf{i}}_{r}(\varphi)$ preserves Theorem 3.1 .

\subsection{The I/E-rules}

By inspecting (3.11) one can realise that Gentzen's positive rules in $N K$, except the I/E-rules for negation, can be carried over to $\mathcal{N}_{\mathbf{i}}$.

For negation, I introduce the following I/E-rules.

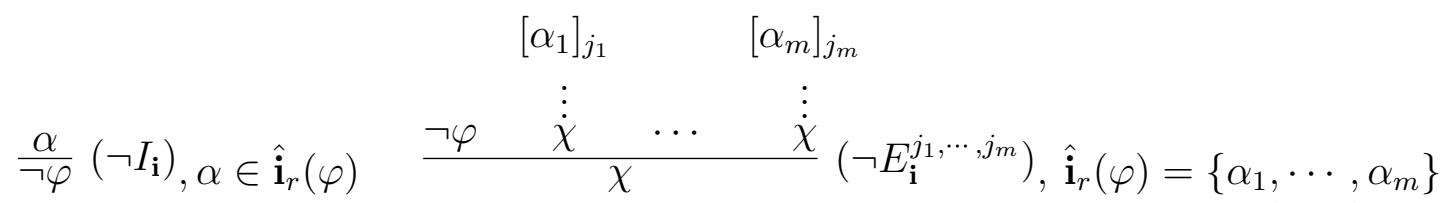

Definition 4.8 (i-consistency) $\Gamma$ is $\mathbf{i}$-consistent iff for no $\varphi$ both $\Gamma \vdash_{\mathcal{N}_{\mathbf{i}}} \varphi$ and $\Gamma \vdash_{\mathcal{N}_{\mathbf{i}}} \neg \varphi$

Theorem 4.3 (soundness of $\mathcal{N}_{\mathbf{i}}$ ) If $\Gamma \vdash_{\mathcal{N}_{\mathbf{i}}} \varphi$ tren $\Gamma \models{ }_{i} \varphi$

Proof. Fix some i. For every rule, I show that if an i-valuation $v$ i-satisfies its premises, then $v$ i-satisfies its conclusion. The claim then follows by induction on the derivation in $\mathcal{N}_{\mathbf{i}}$.

Australasian Journal of Logic (16:5) 2019, Article no. 1 
The only non-standard case is negation.

$\neg I_{\mathrm{i}}$ : Assume that for some $\alpha \in \hat{\mathbf{i}}(\varphi) v \llbracket \alpha \rrbracket=t$. By (3.1), $v \llbracket \varphi \rrbracket=f$. Therefore, by $(3.11) v \llbracket \neg \varphi \rrbracket=t$.

$\neg E_{\mathrm{i}}$ : Assume that $v \llbracket \neg \varphi \rrbracket=t$. By (3.11), $v \llbracket \varphi \rrbracket=f$. By (3.1), there is some $\alpha_{j} \in \mathbf{i}(\varphi)$ s.t. $v \llbracket \alpha_{j} \rrbracket=t$. By the induction hypothesis on the $j$ th minor premise of $\left(\neg E_{\mathbf{i}}\right), v \llbracket \chi \rrbracket=t$.

Theorem 4.4 (completeness of $\mathcal{N}_{\mathbf{i}}$ ) For every atomic incompatibility base i:

$$
\text { If } \Gamma \models{ }_{i} \varphi \text { then } \Gamma \vdash_{\mathcal{N}_{\mathbf{i}}} \varphi
$$

Proof. The proof is almost like the analog proof for propositional classical logic, with one additional important detail. Assuming that $\Gamma={ }_{i} \varphi$ but $\Gamma \nvdash_{\mathcal{N}_{\mathbf{i}}} \varphi$, a counter model is obtained via the Lindenbaum lemma, applied to i-consistency, embedding $\Gamma$ in a maximally-i-consistent set $\hat{\Gamma}$. The additional burden of proof is to show that the valuation $v$ defined by

$$
v \llbracket p \rrbracket=t \text { off } p \in \hat{\Gamma}
$$

extended as usual to

$$
v \llbracket \varphi \rrbracket=t \text { iff } \varphi \in \hat{\Gamma}
$$

is indeed an $\mathbf{i}$-valuation. For that, one needs to establish that if $\varphi \in \hat{\Gamma}$, then $\alpha \notin \hat{\Gamma}$ for every $\alpha \in \hat{\mathbf{i}}(\varphi)$.

Assume, towards a contradiction, that both $\varphi \in \hat{\Gamma}$ and $\alpha \in \hat{\Gamma}$ for some $\alpha \in \hat{\mathbf{i}}(\varphi)$. Then, for some $n \geq 0$, both $\varphi \in \Gamma^{n}$ and $\alpha \in \Gamma^{n}$. But then, both $\Gamma^{n} \vdash_{\mathcal{N}_{\mathbf{i}}} \varphi$ and $\Gamma^{n} \vdash_{\mathcal{N}_{\mathbf{i}}} \neg \varphi$, the latter by applying $\left(\neg I_{\mathbf{i}}\right)$ to $\alpha$, contradicting the i-consistency of $\Gamma^{n}$.

Australasian Journal of Logic (16:5) 2019, Article no. 1 


\section{Atomic incompatibility bases and metaphys- ical grounding}

Metaphysical grounding (henceforth, grounding) is a philosophical notion used for explaining the structure of reality (see [14] for a general introduction). Mostly, it is taken as a relation of dependency of a fact on a set of facts, where a fact is taken as (expressed by) a true proposition. I will conflate the use of those notions and speak mainly about the latter, to remain closer to the level of syntax. Being a dependency relation, grounding is typically considered as a partial ordering over facts.

Because grounding's relata are true propositions, expressed by sentences, it is natural to associate logics with grounds in order to specify the properties of this relation. Two well known logics of grounding are presented in [5] and [3]. A more recent one can be found in [9].

As mentioned in [10], one of the difficulties in devising logics for groundings is the treatment of negation. As noted in [8], both in Fine's logic [5] and in Coreia's logic [3], negation, differently from conjunction and disjunction, is treated only in its interaction with other connectives.

In [9], Poggiolesi proposes an elaborate, rather complicated, logic for grounding, that has also rules for negation. Remarkably, for atomic propositions, Poggiolesi's insight's is very similar to that of the current paper. She notes that the truth of the negated atomic proposition the ball is not cubic can be grounded in the truth of the atomic proposition the ball is spherical, expressed in my terms,

$$
\text { cubic } \in \hat{\mathbf{i}}(\text { spherical) and spherical } \in \hat{\mathbf{i}}(\text { cubic })
$$

(together with the other possible shapes). However, this is not followed by an expansion into a definition of grounding negated compound formulas as I do.

Without attempting here to devise a full logic of grounds, I do propose the following definition for grounds of negated formulas.

Australasian Journal of Logic (16:5) 2019, Article no. 1 
Definition 5.9 (ultimate grounds for negation) The ultimate grounds of $\neg \varphi$, denoted by $\mathbf{g} \llbracket \neg \varphi \rrbracket$, are defined by

$$
\mathbf{g} \llbracket \neg \varphi \rrbracket={ }^{\text {df. }} \hat{\mathbf{i}}_{r}(\varphi)
$$

That is, the grounds of a negated sentence are positive incompatible sentences of a particularly simple form.

For example, the ball is green (all over) is one of the ultimate grounds of the ball is not red (all over).

I refer to $\mathbf{g} \llbracket \neg \varphi \rrbracket$ as 'ultimate ${ }^{5}$ ' because of its constitution only of conjunctions of atomic formulas. In a sense, this notion of grounding is a kind of transitive closure of more common definitions of grounding, grounding negated sentences on "atomic facts" only, the latter conceivable as "building blocks" of reality.

I believe that a general notion of ultimate grounds is worth pursuing for arbitrary truths, not just negated ones. I leave this for further research.

\section{Conclusions}

I presented a plan for negation, proposing a paradigm shift from the Australian plan, leading to a family of contra-classical logics $\mathcal{L}_{\mathbf{i}}$. The two main ideas are the following:

1. Instead of shifting points of evaluation (in a frame), shift the evaluated formula.

2. Introduce an incompatibility set for every atomic formula, extended to any compound formula, and impose the condition on valuations that a formula evaluates to true iff all the formulas in its incompatibility set evaluate to false. Thus, atomic sentences are not independent in their truth-values.

\footnotetext{
5This notion can be thought of as an opposite pf Poggiolesi's 'immediate grounds'.
}

Australasian Journal of Logic (16:5) 2019, Article no. 1 
I have also presented a sound and complete natural deduction proof system $\mathcal{N}_{\mathbf{i}}$ for every $\mathcal{L}_{\mathbf{i}}$.

In addition, the kind of negation considered in this paper is shown to provide an innovative notion of grounding negation.

By imposing other (than incompatibility) relations among atomic formulas

as restricting valuation, other families of logics can be obtained. I pursue elsewhere variations on this theme.

What about paraconsistency and paracompleteness?

I believe the right way to obtain them is to change the definition of logical consequence, not of the meanings of the connectives. Adopting Priest's null account in [11] leads to the following definition of logical consequence, both paraconsistent and paracomplete:

For a given atomic incompatibility base $\mathbf{i}, \psi$ is a (logical) $\mathbf{i}$-consequence of $\Gamma$, denoted $\Gamma={ }_{\mathbf{i}} \varphi$, iff:

1. $\Gamma$ is $\mathbf{i}$-consistent, and

2. $\neg \psi$ is $\mathbf{i}$-consistent, and

3. for every i-valuation $v: v \llbracket \psi \rrbracket=t$ whenever $v \llbracket \varphi \rrbracket=t$ for every $\varphi \in \Gamma$.

\section{Acknowledgments}

I thank Michael Kaminski for useful discussions on the topic of the paper and critical comments on a preliminary draft, in spite of not agreeing with the proposed plan.

Australasian Journal of Logic (16:5) 2019, Article no. 1 


\section{References}

[1] Francesco Berto and Greg Restall. Negation on the Australian plan. Journal of Philosophical Logic (forthcoming), 2018.

[2] Robert Brandom. Between saying and doing: towards an analytic pragmatism. Oxford University Press, Oxford, UK., 2001.

[3] Fabrice Correia. Logical grounds. Review of Symbolic Logic, 7:31-59, 2014.

[4] Michael De and Hitoshi Omori. There is more to negation than modality. Journal of Philosophical Logic, 47(2):281-299, 2018.

[5] Kit Fine. Guide to ground. In Fabrice Correia and Benjamin Schnieder, editors, Metaphysical grounding, pages 37-80. Cambridge University Press, Cambridge, 2012.

[6] Germán Kruszewski, Denis Papernoand, Raffaella Bernardi, and Marco Baroni. There is no logical negation here, but there are alternatives: Modeling conversational negation with distributional semantics. Computational Linguistics, 42(4):637-660, 2016.

[7] Sara Negri. Proof theory for modal logic. Philosophy Compass, 6/8:523$538,2011$.

[8] Francesca Poggiolesi. A critical overview of the most recent logics of grounding. In Francesca Boccuni and Andrea Sereni, editors, Objectivity, Realism, and Proof. Springer, Cham, 2016. Boston Studies in the Philosophy and History of Science, vol 318.

[9] Francesca Poggiolesi. On constructing a logic for the notion of complete and immediate formal grounding. Synthese (forthcoming), 2016. DOI: 10.1007/s11229-016-1265-z.

[10] Francesca Poggiolesi. Bolzano, (the appropriate) relevant logic and ground-ing rules for implication. In Benjamin Schnieder and Sefan Roski., editors, Bolzano and grounding. Oxford University Press, Oxford, UK, 2018.

Australasian Journal of Logic (16:5) 2019, Article no. 1 
[11] Graham Priest. Negation as cancellation, and connexive logic. Topoi, 18(2):141-148, 1999.

[12] Stephen Read. Semantic pollution and syntactic purity. Review of symbolic logic, 8(4):649-661, 2015.

[13] Greg Restall. Defining double negation elimination. Logic Journal of the IGPL, 8(6):853-860, 2018.

[14] Kelly Trogdon. An introduction to grounding. In Miguel Hoeltje, Benjamin Schnieder, and Alex Steinberg, editors, Dependence. Basic Philosophical Concepts, pages 97-122. Philosophia Verlag, 2013.

Australasian Journal of Logic (16:5) 2019, Article no. 1 